\title{
Research on Wind Power System with Adaptive Speed Control of MPPT Algorithm
}

\author{
Ai-mei Xiao and Yong-xin Jia \\ 1. Department of Computer Science and Technology, Shandong University of Technology, Zibo 255049, Shandong Province, China
}

\begin{abstract}
MPPT (Maximum Power Point Tracking) is one of the most important technologies in the wind power system. It analyses the wind power system's model. Based on properties of wind machine's mechanical and electrical, around the rotor dynamics and the excitation winding voltage, this paper researches the automatic tracking algorithm of rotational speed. By dynamically changing winding voltage it controls the generator reaction torque, then the rotational speed is adjusted and it develops a new nonlinear adaptive transmission control model for wind power generator. This control model can adjust the excitation winding of the alternator voltage dynamically and make wind turbines to achieve the maximum power point tracking rapidly.
\end{abstract}

Key words: Adaptive gearshift, maximum power point tracking, excitation voltage, rotor dynamics, speed tracking.

\section{Introduction}

As a pollution-free renewable resource, wind power has the advantages such as non-pollution, inexhaustible supply, etc. It plays an important position in the future power supply system, and it is also an important symbol of development and progress of human society in the 21st century. But the problem existing in the wind power generation system is that the actual power output is greatly influenced by external factors, and there is a wide gap between the actual power output and the predicted power output [1]. Most of the existing wind turbines adopt the constant speed operation mode, that is, the speed of the wind turbine does not change along with the change of wind speed, therefore, the utilization efficiency of wind energy is low. The variable speed wind turbine, with its advantage of being able to trap maximum wind energy, has gained attention from the scholars at home and abroad, and its installed capacity is increasing every day. It can make the wind wheel's rotational speed rapidly track the change of wind speed, which makes the wind power generator always keep running at the optimum tip speed ratio, thereby

Corresponding author: Xiao Ai-Mei, lecturer, research fields: algorithm and network information security. the wind energy is obtained to the maximum extent [2].

Since the wind power generation was put into practical use, many MPPT (maximum power point tracking) algorithms [3] have been developed, and there have been also some theoretical researches about conversion efficiency. To make full use of wind power, starting from analyzing the characteristics of the wind turbine, it needs to capture the maximum power point of the wind power system and realize automatic tracking. Due to the randomness of the wind speed and nonlinearity of the wind power system, it is quite difficult to control and capture the largest capacity point, which is also one of the hot issues of wind power [4].

The paper is organized as follows: Section 2 explains model introduction; Section 3 discusses establishment of the automatic control model; Section 4 contains the study of simulation; Section 5 is the conclusion.

\section{Model Introduction}

According Baez theory, the mechanical power output of a wind turbine can be written as [5]:

$$
P_{m}\left(V_{w}\right)=\frac{1}{2} C_{p}(\lambda, \beta) \rho \pi R^{2} V_{w}^{3}
$$


$\rho$ is the air density, $R$ is the radius of the turbine shaft, $C_{P}$ is the power coefficient (dimensionless), $V_{w}$ is the wind velocity.

Under a certain wind speed, the input mechanical power acquired by the generator only depends on wind energy conversion coefficient [6], which in turn is the function between tip speed ratio and blade pitch angle 4. Under the condition that the wind speed is definite, input mechanical capacity the generator obtained will be only determined by wind energy conversion coefficient [7].

In Eq. (1), the power which the generator obtains from wind is in direct proportion to capacity coefficient and the cubic of wind speed. When is definite, the relation between and shows a parabolic relation [8]. Fig. 1 shows the relation curve between them. The salient point corresponds to the capture point of the maximum wind energy, which is called the optimum tip speed ratio here. And it reaches the maximum for the time being. Because of the random variation characteristic of the wind speed, it often cannot work on the maximum point. Instead of it is in a status of low conversion efficiency. If the method of adjusting the blade pitch angle to adjust the running of the wind turbine is adopted, then it belongs to the mechanical adjustment. In general, for adopting the electrical adjustment, the blade pitch angle is a constant. Therefore, is just the function of, and the relation curve between them is also one of the basic characteristics of the wind turbine.
The tip speed ratio is the function of the ratio of the wind wheel tip linear speed to the wind speed:

$$
\lambda=\frac{w R}{V_{w}}
$$

Where, $w$ is the rotor speed (rad/s), $V_{w}$ is the wind velocity ( $\mathrm{r} / \mathrm{min})$.

A typical relationship between $C_{P}$ and $\lambda$ is shown in Fig. 1

From Eqs. (1) and (2), it can be concluded that

$$
P_{m}\left(V_{w}\right)=k_{w} w^{3}
$$

Where:

$$
k_{w}=\frac{1}{2} C_{p} \rho \pi \frac{R^{5}}{\lambda^{3}} .
$$

The block diagram of the wind turbine system in Fig. 2 shows how the wind turbine works. The wind energy conversion system includes a wind turbine that turns wind energy into mechanical energy, and a gear box which can greatly increase the rotating speed of the generator. The generator turns the mechanical energy which is converted by wind energy into electric energy. According to the mechanical properties of the wind turbine, the following equations can be easily obtained:

$$
\begin{gathered}
T_{m}-T=J_{m} \dot{w} \\
T_{p}-T_{e}=J_{e} \dot{w}_{e} \\
T_{p} w_{e}=T w
\end{gathered}
$$

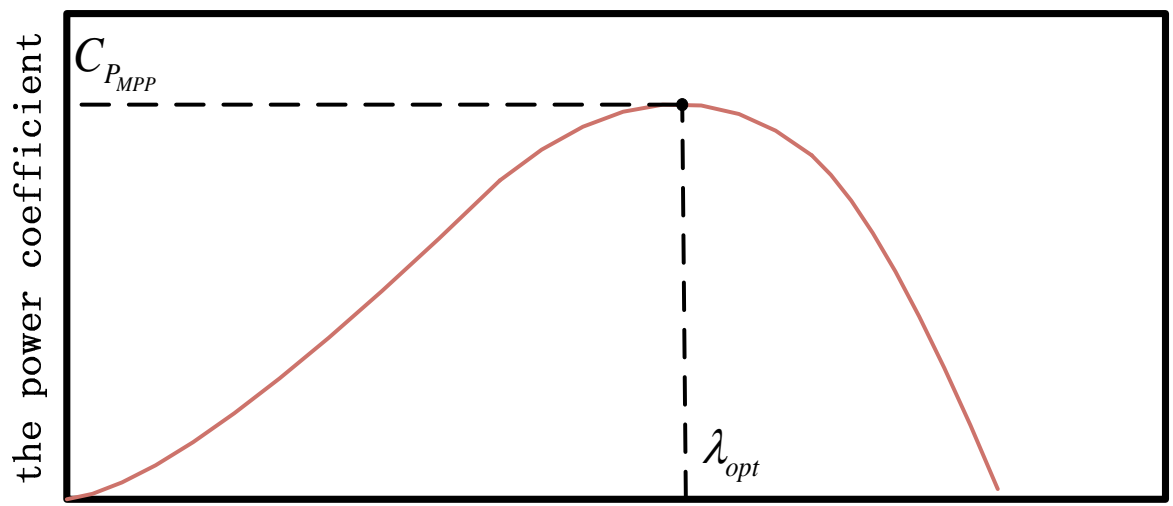

The tip speed ratio

Fig. 1 Typical power coefficient curve. 


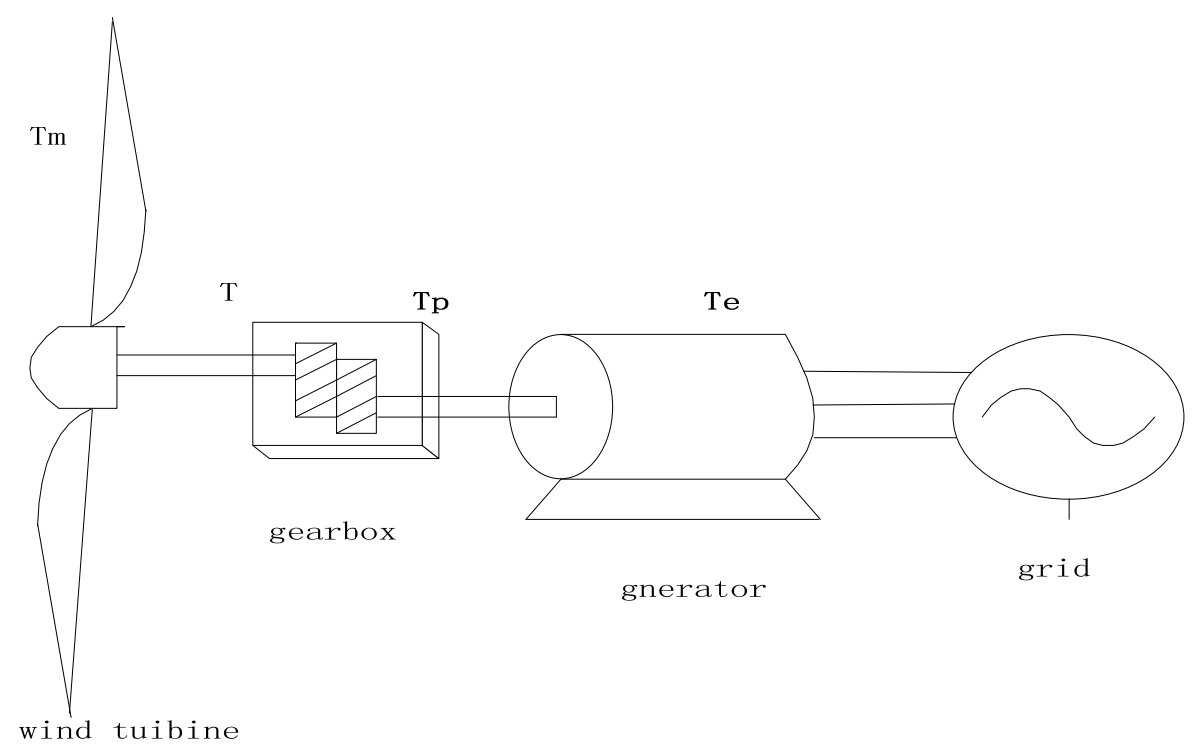

Fig. 2 Block diagram of the wind turbine system.

$T_{m}$ is the wind torque

$T$ is the transmission input torque;

$T_{p}$ is the transmission output torque;

$T_{e}$ is the a shaft torque;

$w_{e}$ is the generator angular velocity;

$J_{m}$ is the moment of inertia of the turbine;

$J_{e}$ is the moment of inertia of the generator.

We defined the gear ratio $G$

$$
G=\frac{w_{e}}{w}
$$

Using Eqs. (6) and (7), integrated Eqs. (4) and (5), it can be concluded that

$$
J \dot{W}=T_{m}-G T_{e},
$$

Rearranging $J=J_{m}+G^{2} J_{e}$ results in Eq. (8)

$$
J \dot{w}=\frac{P_{m}}{w}-G \frac{P_{e}}{w_{e}}
$$

$P_{m}$ is the wind power, $P_{e}$ is the electric power.

$$
P_{e}=K_{\phi} w_{e} c\left(I_{f}\right)
$$

Where $K_{\phi}$ is a machine-related constant, $c\left(I_{f}\right)$ is the flux in the generating system, $I_{f}$ is the field current. Then the exciter dynamics of the system is governed.

$$
L \dot{I}_{f}+I_{f} R_{f}=u_{f}
$$

Where $L$ is the inductance of the circuit, $R_{f}$ is the field current, $u_{f}$ is the field voltage.

\section{Establishment of the Automatic Control Model}

To achieve a better control performance, both rotor dynamics Eq. (8) and excitation dynamics Eq. (10) must be taken into consideration. The rotor speed of the wind turbine is controlled by adjusting the excitation winding voltage. In the algorithm design, it changes the winding voltage to control the reaction torque of the generator, and then makes the rotation rate get relevant adjustment. Therefore the control method of adaptive variable speed control is concluded: A control voltage is designed to enable the rotor speed of the wind turbine to track the designed speed closely. Suppose there is a limit for, and Eqs. (8) and (10) are rewritten to obtain:

$$
\dot{w}=A \Phi-B c\left(I_{f}\right)
$$

and

$$
\dot{I}_{f}=b u_{f}-a I_{f}
$$

where

$$
\begin{aligned}
& A=\frac{k_{w}}{J}, \quad B=\frac{G K_{\phi}}{J}, \\
& \Phi=w^{2}, \\
& a=\frac{R_{f}}{L}, \quad b=\frac{1}{L} .
\end{aligned}
$$


To design the tracking controller, let us define the rotor tracking error as:

$$
e=w-w^{*}
$$

Differentiating upon Eqs with respect to and rearranging it results in:

$$
\dot{e}=A \Phi-B c\left(I_{f}\right)-\dot{w}^{*}
$$

Now we consider that the parameters such as $k_{w}$, $J$ and $A$ are unknown, we define be the estimate of and be the estimate error defined by then we rewrite the error dynamic equation as:

$$
\dot{e}=-k_{0} e+z_{a}+\tilde{A} \Phi
$$

where

$$
z_{a}=\hat{A} \Phi-B c\left(I_{f}\right)-\dot{w}^{*}+k_{0} e
$$

$k_{0}>0$ and it is a constant. In the Eq. (15), if $z_{a}$ is made to approach zero as $t \rightarrow \infty$, then with $t \rightarrow \infty$ we can get that $e \rightarrow 0$. Then what we need to do is to making $Z_{a} \rightarrow 0$. Now we take derivative of the Eq. (15) and substituting for $\dot{w}, \dot{e}$ and $\dot{I}_{f}$, then we get the Eq. (16)

$$
\begin{aligned}
\dot{z}_{a}= & \dot{\hat{A}} \Phi+\hat{A} \frac{\partial \Phi}{\partial w} \dot{w}-B \frac{\partial c}{\partial I_{f}} \dot{I}_{f}-\ddot{w}^{*}+k_{0} \dot{e} \\
= & \dot{\hat{A}} \Phi+\hat{A} \frac{\partial \Phi}{\partial w}\left(\dot{e}+\dot{w}^{*}\right)-B \frac{\partial c}{\partial I_{f}} \dot{I}_{f}-\ddot{w}^{*}+k_{0} \dot{e} \\
= & \dot{\hat{A}} \Phi+\left(\hat{A} \frac{\partial \Phi}{\partial w}+k_{0}\right)\left(-k_{0} e+z_{a}+\tilde{A} \Phi\right) \\
& -B \frac{\partial c}{\partial I_{f}} b u_{f}+B \frac{\partial c}{\partial I_{f}} a I_{f}+\hat{A} \frac{\partial \Phi}{\partial w} \dot{w}^{*}-\ddot{w}^{*} \\
= & F_{a}-b_{a} u_{f}+\left(\hat{A} \frac{\partial \Phi}{\partial w}+k_{0}\right) \tilde{A} \Phi
\end{aligned}
$$

Where

$$
\begin{gathered}
F_{a}=\dot{\hat{A}} \Phi+\left(\hat{A} \frac{\partial \Phi}{\partial w}+k_{0}\right)\left(-k_{0} e+z_{a}\right) \\
+B \frac{\partial c}{\partial I_{f}} a I_{f}+\hat{A} \frac{\partial \Phi}{\partial w} \dot{w}^{*}-\ddot{w}^{*} \\
b_{a}=B \frac{\partial c}{\partial I_{f}} b .
\end{gathered}
$$

Now if we design the control voltage so that

$$
u_{f}=\frac{1}{b_{a}}\left(F_{a}+k_{a} z_{a}\right) .
$$

the Eq. (16) is rewrited as:

$$
\dot{z}_{a}=-k_{a} z_{a}+\left(\hat{A} \frac{\partial \Phi}{\partial w}+k_{0}\right) \tilde{A} \Phi .
$$

Consider the Lyapunov function candidate

$$
V=\frac{1}{2} e^{2}+\frac{1}{2} z_{a}^{2}+\frac{1}{2} \tilde{A}^{2}
$$

Differentiating $V$, then

$$
\begin{aligned}
\dot{V}= & e \dot{e}+z_{a} \dot{z}_{a}-\tilde{A} \dot{\hat{A}} \\
= & e\left(-k_{0} e+z_{a}+\tilde{A} \Phi\right)+ \\
& z_{a}\left[-k_{a} z_{a}+\left(\hat{A} \frac{\partial \Phi}{\partial w}+k_{0}\right) \tilde{A} \Phi\right]-\tilde{A} \dot{\hat{A}} \\
& =-k_{0} e^{2}-k_{a} z_{a}^{2}+e z_{a}+ \\
& \tilde{A}\left[e \Phi+z_{a}\left(\hat{A} \frac{\partial \Phi}{\partial w}+k_{0}\right) \Phi-\dot{\hat{A}}\right]
\end{aligned}
$$

If the estimate parameters $\dot{\hat{A}}$ are updated via

$$
\dot{\hat{A}}=e \Phi+z_{a}\left(\hat{A} \frac{\partial \Phi}{\partial w}+k_{0}\right) \Phi .
$$

we can get the Eq. (23)

$$
\begin{aligned}
\dot{V} & =-k_{0} e^{2}-k_{a} z_{a}^{2}+e z_{a} \\
& =\left(\begin{array}{ll}
e & z_{a}
\end{array}\right)\left(\begin{array}{cc}
-k_{0} & 1 \\
0 & -k_{a}
\end{array}\right)\left(\begin{array}{l}
e \\
z_{a}
\end{array}\right) \\
& \leq-\lambda_{\min }\left(\begin{array}{ll}
k_{0} & k_{a}
\end{array}\right)\left(e^{2}+z_{a}^{2}\right) \leq 0 .
\end{aligned}
$$

and then we have

$$
e \in L_{\infty} \cap L_{2}, z_{a} \in L_{\infty} \cap L_{2} \quad \text { and } \quad \tilde{A} \in L_{\infty} .
$$

From the Eq. (15) we see that the boundedness of $z_{a}, e$ and $\hat{A}$ ensures that $\hat{A} \Phi$ is bounded. So $\Phi \in L_{\infty}$, then $\tilde{A} \Phi \in L_{\infty}$, At last we can get $\dot{e} \in L_{\infty}$. According to Barbalat lemma, we can conclude that both $e$ and $z_{a}$ converge to zero asymptotically and $\hat{A}$ remain bounded. Thus the following result is established. Consider the wind power generation system given by Eq. (8) with the field exciter as shown in Eq. (10). If the field voltage is adjusted according to Eq. (19), where is generated by Eq. (15) and $\hat{A}$ are updated by Eq. (23), then the rotor speed tracks the desired speed asymptotically. 


\section{Simulation Study}

To verify the effect of the adaptive tracking control strategy, the following simulation work was carried out. The selection of some control parameters was as follows:

$$
k_{a}=45, \quad k_{0}=1.147 .
$$

In the simulation experiment, the ideal trajectory to be tracked was selected as:

$$
w^{*}=3+2 \times \sin (t) \text {. }
$$

Fig. 3 shows a comparison diagram between the actual and the ideal rotation speed, and the red line represents the curve of the ideal rotation speed, while the yellow line represents the curve of the actual rotor speed. The figure shows that the actual rotation speed can quickly track the ideal design curve and can also keep a highly accurate tracking.

Fig. 4 shows the tracking error curve. In a short response time, it realizes a tracking with almost zero error. Obviously, the controller designed makes use of the adaptive speed control method to complete rotational speed tracking efficiently and smoothly. The control voltage is depicted in Figs. 5 and Fig. 6 shows the estimate parameters. It is observed that the proposed method achieves the smooth and effective tracking.

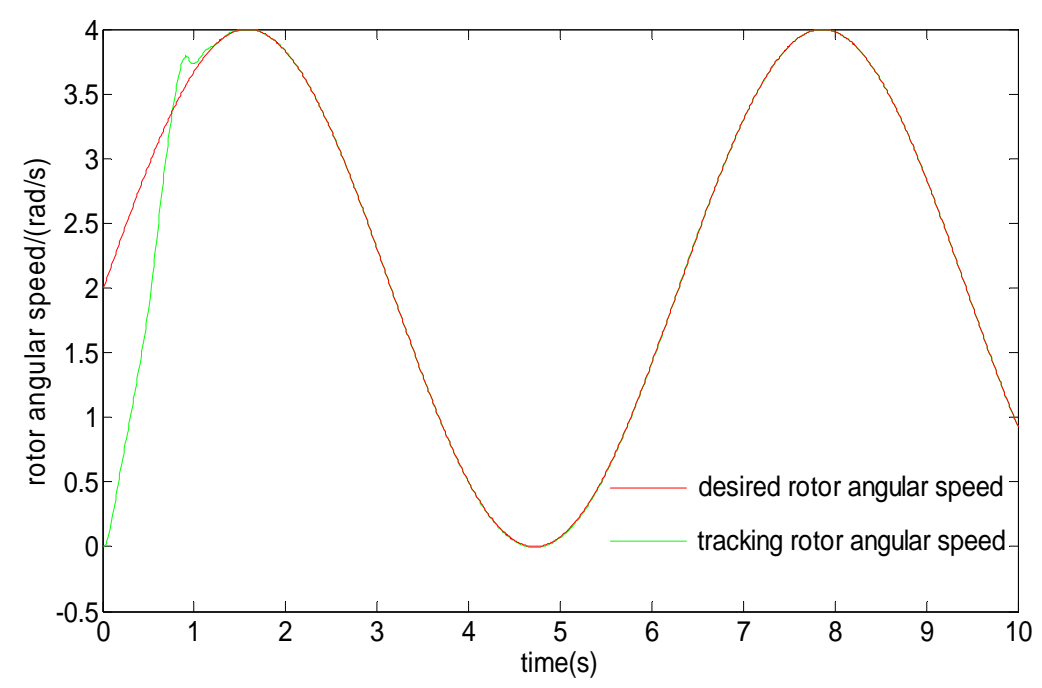

Fig. 3 Tracking of rotor angular speed.

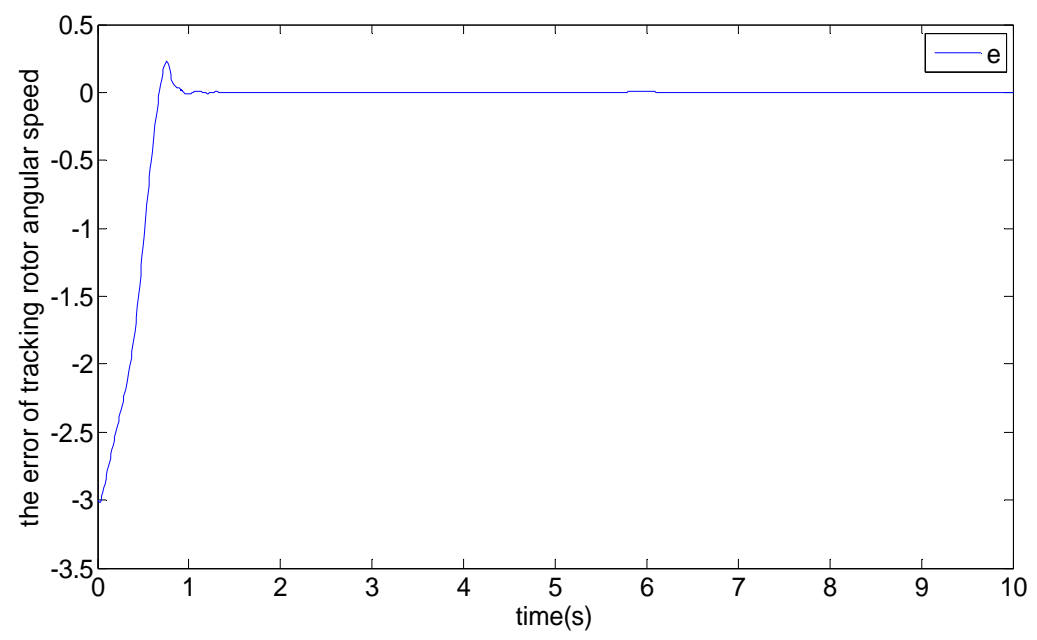

Fig. 4 Tracking error. 


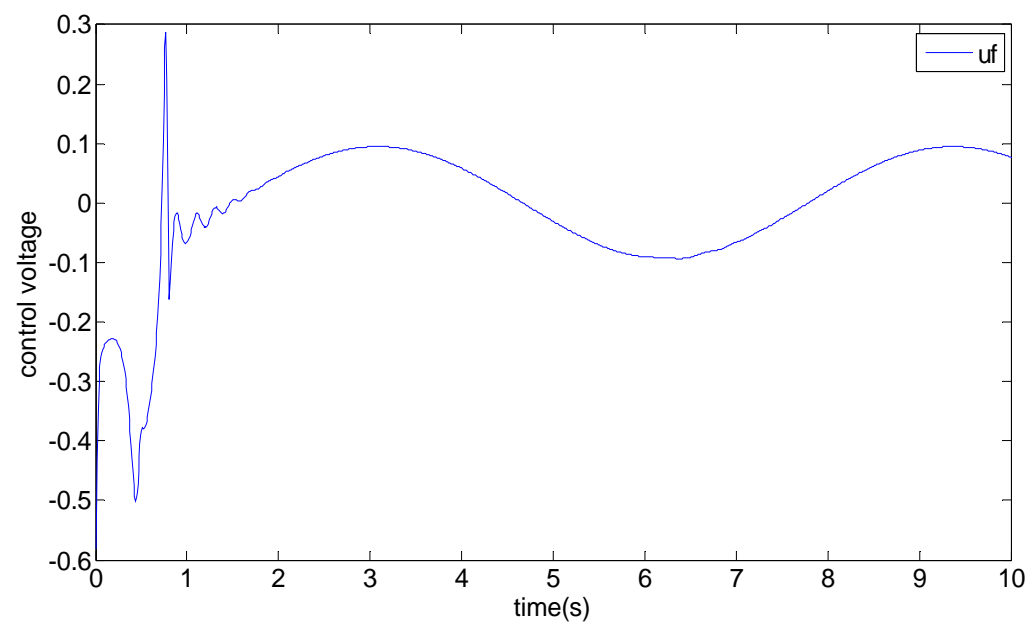

Fig. 5 Control voltage.

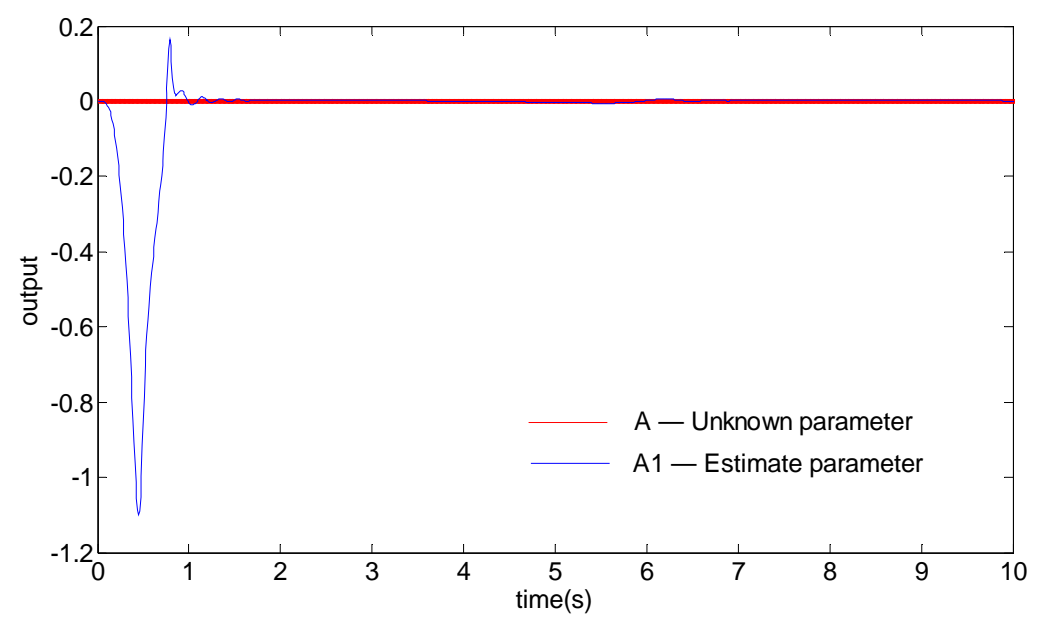

Fig. 6 Estimation of unknown parameter.

\section{Conclusion}

With the maturing of wind power technology, the continuous improvement of the quality of wind power products and the gradual decrease of the cost of wind power, wind power already has the potential for large-scale development. Due to the nonlinearity of the wind power system, changes of environmental conditions, and influence of other factors, the MPPT method has become a complex and comprehensive problem.

Apart from considering the intrinsic characteristics of each method, the selection of the MPPT method also needs to consider several factors of the controlling method, such as the difficulty, economic costs, sensor type, tracking speed and accuracy, application areas. It adopts an intelligent way to track the largest capacity point to realize the maximum utilization of the wind energy. The simulation result proves that the adopted control strategy can complete the capture of the largest capacity under different speeds of wind, which offers a better comprehensive benefit.

\section{References}

[1] Abdullah, M. A., Yatim, A. H. M., Tan, C. W., and Saidur, R. 2012. A Review of Maximum Power Point Tracking Algorithms for Wind Energy Systems.” Renewable and Sustainable Energy Reviews 16 (5): 3220-7.

[2] Freris, L. L. 1990. Wind Energy Conversion Systems. Englewood Cliffs, NJ: Prentice-Hall.

[3] Bharanikumar, R., Yazhini, A. C., and Kumar, A. N. 2005. "Novel Maximum Power Point Tracking Controller for Wind Turbine Driven Permanent Magnet Generator.” IEEE Transaction 20 (2). 
[4] Blaabjerg, F., Chan, Z., and Kjaer, S. B. 2004. "Power Electronics as Efficient Interface Indispersed Power Generation Systems.” IEEE Trans. Power Electron 19 (5): 1184-94.

[5] Barakati, S. M., Kazerani, M., and Chen, X. 2005. “A New Wind Turbin Egeneration System Based on Matrix Converter.” In Proc. IEEE/PESGeneral Meeting, 2083-9.

[6] Neammanee, B., and Krajangpan, K. 2007. "Maximum Peak Power Tracking-Based Control Algorithms with
Stall Regulation for Optimal Wind Energy Capture.” In Power Conversion Conference, 1424-30.

[7] Wang, Q., and Chang, L. C. 2004. “An Intelligent Maximum Power Extraction Algorithm for Inverter-Based Variable Speed Wind Turbine Systems.” IEEE Transactions on Power Electronics 19 (5): 1242-49.

[8] Jouko, N., Slavomir, S., and Jari, P. M. 2010. Low Voltage Ride through Testing of Wind Turbine Drives [EB/OL] .http: / /www.abb.com/windpower. 